\title{
UNUSUAL BEHAVIOR IN CHILDREN- FOREIGN OBJECT IN TOOTH: A REPORT OF TWO CASES
}

\author{
Mahesh $\mathbf{R}^{1}$, Waseem $\mathbf{H}^{2}$, Siva kumar $\mathbf{M}^{3}$
}

\begin{abstract}
BACKGROUND: The chance of discovering foreign object in a root canal is more commonly seen in children. Foreign objects may act as a potential source of infection, which may hinder the complete debridement of root canal. Although the main etiology being an accidental insertion, there are other possibilities such as self-injurious habits which should be ruled out.

CASE DETAILS: The present article describes two case of foreign object in root canal, their possible etiology and management modalities. The first case reports a usual case of staple pin in root canal of primary teeth. The second case was a broken pin inside anterior permanent teeth. The cases reported highlight the need for proper diagnosis and early management.

CONCLUSION: This case report highlights the need for proper understanding of the etiology in management of foreign body in root canal system.
\end{abstract}

KEY WORDS: Foreign object, Self-Embedding habit, Stapler pin

DOI: http://dx.doi.org/10.4314/ejhs.v24i4.14

\section{INTRODUCTION}

Endodontic management of an infected root canal can be complicated by the presence of foreign object. The foreign body may hinder the complete debridement of root canal and can also act as a potential source of infection. The chance of foreign body is more commonly seen in children, who have a tendency to swallow or insert small instruments while playing or it can be an accidental.

The chance of foreign object getting impacted in the teeth is more common when pulp chamber is open either because of traumatic injury or large carious exposure (1). In most of the situations, the foreign objects are diagnosed in routine radiographs, which play a vital role in analyzing the location, size and type of the foreign object. The management protocol depends on the location of the foreign object, the type of dentition and the stage of root formation. While the main etiology is an accidental insertion of foreign objects, there are other possibilities such as self-injurious habits which should be ruled out mostly in children. This article describes two cases of foreign object impacted in the root canal, the possible etiology and the various treatment protocols.

\section{CASE REPORTS}

Case 1

A 5 years old boy reported with a chief compliant of pain and swelling in the upper left front tooth. The patient expressed a history of chronic discharging sinus in relation to the upper right maxillary primary incisor (51) for the past 6 months. Clinical examination revealed caries with open pulp chamber in relation to 51 (Fig 1). Radiograph revealed a radio-opaque object which resembles a staple pin in the root canal space of 51 , associated with peri apical radiolucency as can be seen in Fig 2. The parent gave a history that the child used to put sharp items such as pins or

\footnotetext{
${ }^{1}$ Department of Pediatric Dentistry, Saveetha Dental College and Hospitals, Chennai, India.

${ }^{2}$ Department of Pediatric Dentistry, Priyadharshini Dental College and Hospitals, Chennai, India.

${ }^{3}$ Department of oral pathology, Madha Dental College and Hospitals, Chennai, India.

Corresponding Author: Mahesh R, Email: maheshpedo@gmail.com
} 
sticks in his upper front tooth. Considering the prognosis of the teeth and associated peri apical lesion, it was decided to extract the central incisor (51) (Fig 3).

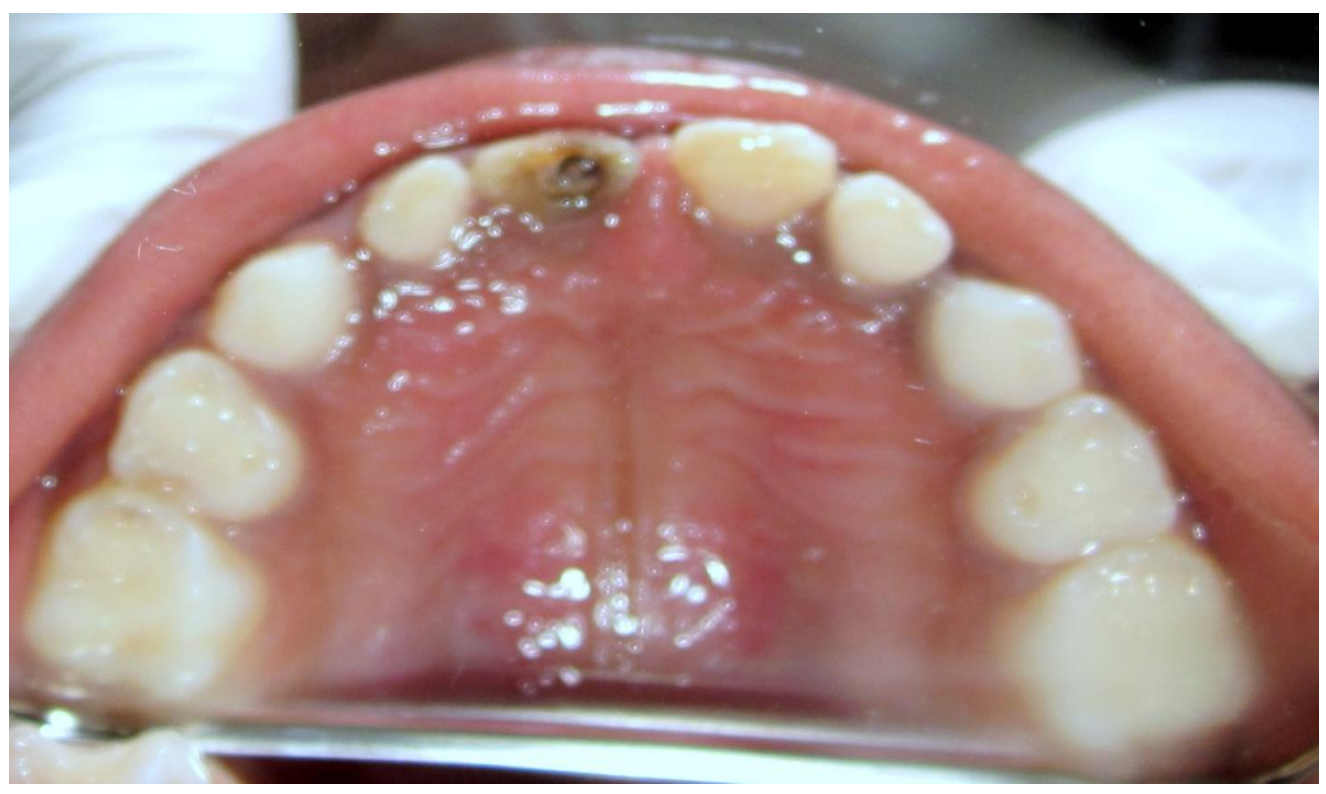

Fig.1: Intra oral photograph showing decayed 51

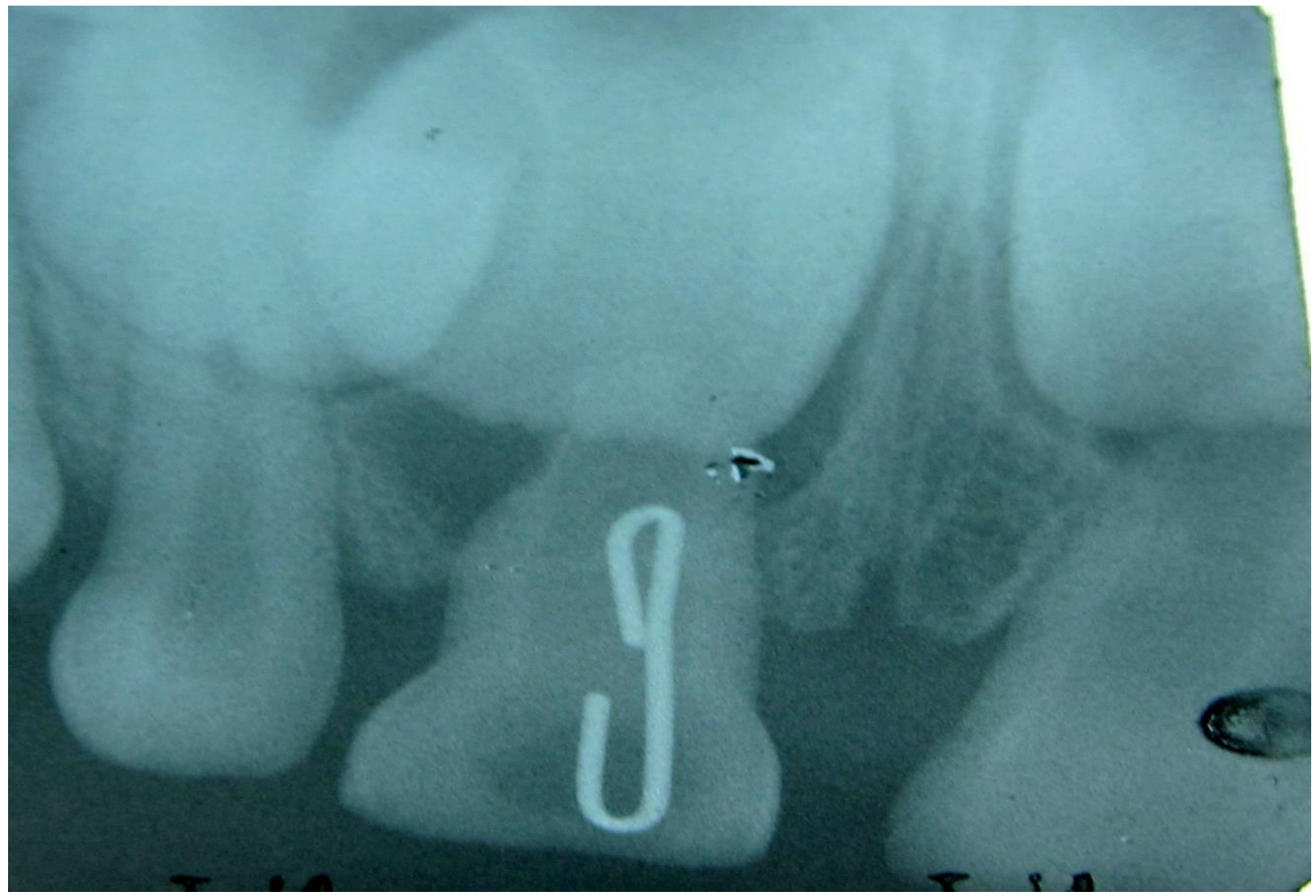

Fig.2: Radiograph showing the foreign object in the root canal 


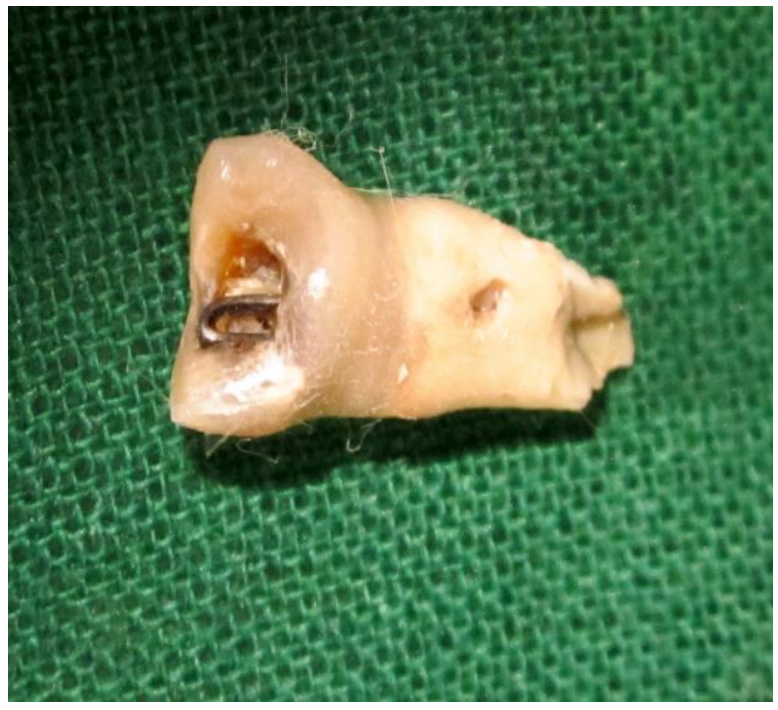

Fig. 3: Photograph showing extracted 51
An 11 years old boy reported with a history of broken front tooth in a trauma while playing 10 months before presentation. On intra oral examination, there was discolouration in the upper left central incisor (21) with opened pulp chamber (Fig 4). Radiograph revealed a radio-opaque object in the root canal space of 21 (Fig 5). The patient gave a history of placing pencil and pin hooks in the upper front tooth to relieve chronic irritation. The access was opened under rubber dam and the object was retrieved using an ISO no 25 Hedstroem File (Dentsply). On removal, the foreign object was found to be a broken metal pin (Fig 6). The canal was sterilized using non setting calcium hydroxide [pure calcium hydroxide, Biopulp, Calcicure] and obturated with thermo filled gutter percha (Thermafil, Dentsply).

\section{Case 2}

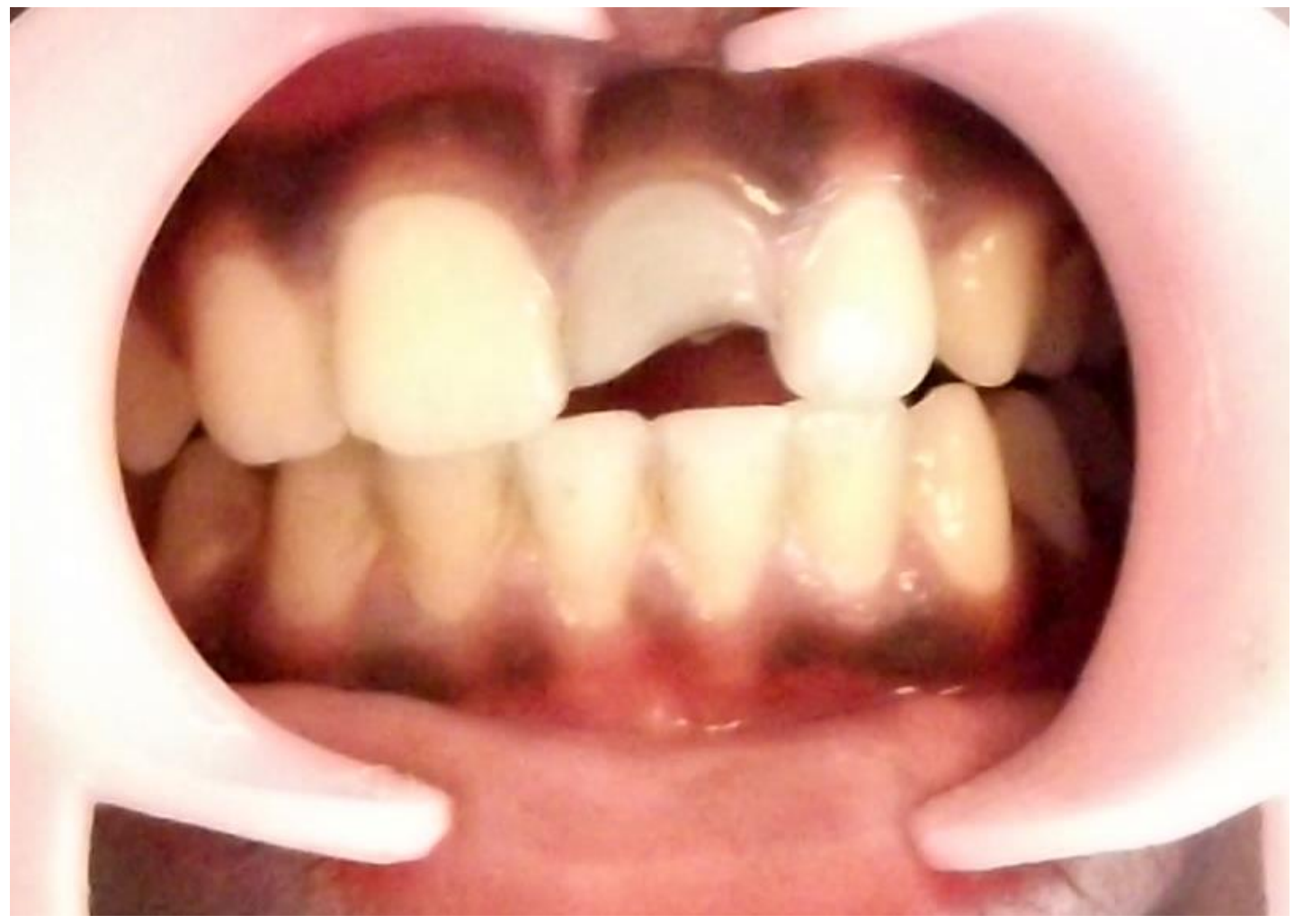

Fig.4: Intra oral photograph showing fracture with open root canal in 21 


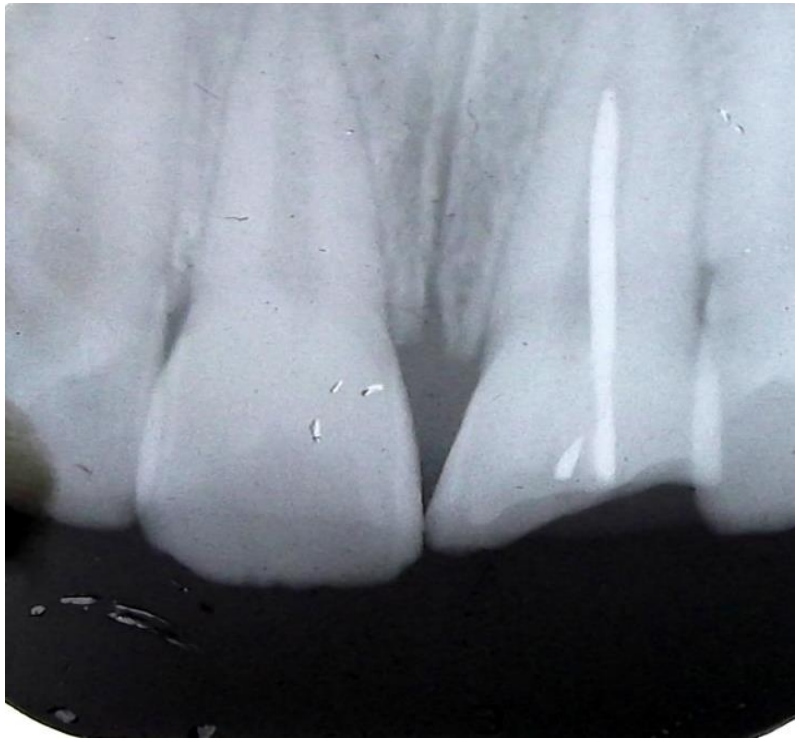

Fig. 5: Peri apical radiograph showing the presence of broken metallic pin.

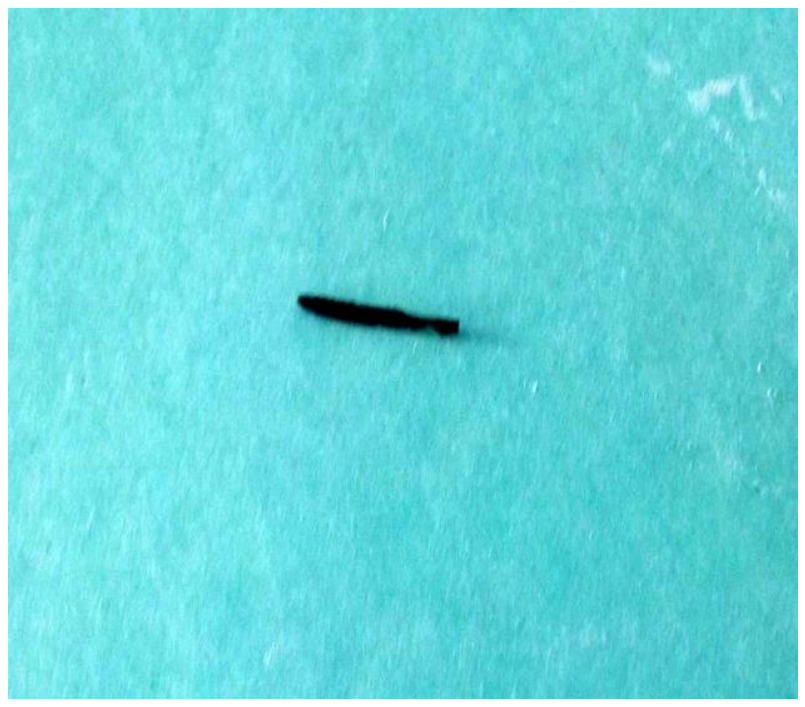

Fig. 6: Foreign object retrieved from the root canal

\section{DISCUSSION}

Root canals can be blocked by the presence of broken instruments, canal obturation materials and in some cases by foreign objects inserted by the own patients (2). Foreign objects in the root canals should be carefully evaluated to determine their nature, position, size and the degree of difficulty that maybe encountered during retrieval (3). McAuliffe et al (2005) summarized various radiographic methods to be followed to localize a radio opaque foreign object as Parallax views, Vertex occlusal view, Triangulation techniques, Stereo Radiography and Tomography (4).

Literature review shows that these incidences are more commonly reported in children, who had a tendency to keep foreign objects in the mouth especially while studying and/or watching T.V (5). In pediatric dentistry, the foreign objects are more commonly diagnosed only in a routine radio graphic examination. The important reason is the fear of punishment towards the parent and unawareness by the children (6).

In the first case, the child had a habit of placing pins to relieve his chronic symptoms, which is mainly due to untreated dental problems. The deciduous tooth was extracted because of the possibility of any potential damage to the permanent teeth.

The second case indicates the need for careful management techniques in removal of any foreign object from the root canal space without a need for peri-apical surgery. Hedstroem Files provide a much easier and convenient technique in engaging any foreign objects in the root canal. Foreign object retrieval and calcium hydroxide dressing can help to eliminate the chronic periapical infection.

Younger children usually swallow or insert foreign objects accidentally, usually as a result of play or exploring their environment. Adolescents, however, are more likely to swallow or insert foreign objects intentionally as a risk-taking behavior, a bid for attention, or under the influence of drugs or alcohol. These kinds of behavior problems are commonly categorized as self- injurious behavior (7).

Self-injury refers to a broad class of experiences (suicidal and nonsuicidal) in which people engage in behavior that directly and deliberately destroys one's own body tissue (8). Often misdiagnosed, ignored, or underreported, self-embedding behavior (SEB) is a form of selfinjurious behavior involving the insertion of inanimate objects into the soft tissues, either under the skin or into muscles (9). The present case report is not suggestive of any self-injurious habits, the possibilities of any such habit should be ruled out in any case of foreign object in children. Awareness of SEB is paramount for rapid and effective identification, assessment and interruption of the cycle of self-injury. 
Although there are case reports of foreign objects retrieved from root canal, the management protocol differs in each situation (10). This case report signifies the importance of early management of dental problems and awareness creation about self-injurious habits to prevent any long term complications.

\section{REFERENCES}

1. Kalyan SR, Sajjan G. Endodontic management of a foreign body. Contemp Clin Dent, 2010; 1:180-2.

2. Balto H. A radiopaque object in the periradicular area. Egypt Dent J, 2002; 48:1023-6.

3. Prabhakar AR, Namineni S, Subhadra HN. Foreign body in the apical portion of a root canal in a tooth with an immature apex: a case report. Int Endod J, 2008; 41:920-7.

4. McAuliffe N, Drage NA, Hunter B. Staple diet: a foreign body in a tooth. Int J Paediatr Dent, 2005; 15:468-71.
5. Chand K, Joseph S, Varughese JM, Nair MG, Prasanth S. Endodontic management of an unusual foreign body in a maxillary central incisor. J Conserv Dent, 2013; 16:474-6.

6. Lehl G. Foreign body in a deciduous incisor: a radiological revelation. J Indian Soc Pedod Prev Dent, 2010; 28:45-6.

7. Muehlenkamp JJ. Self-injurious behavior as a separate clinical syndrome. Am $J$ Orthopsychiatry, 2005; 75:324-33.

8. Nock MK. Why do people hurt themselves? New insights into the nature and functions of self-injury. Curr Dir Psychol Sci, 2009; 18:78-83.

9. Bennett GH, Shiels WE, Young AS, Lofthouse N, Mihalov L. Self-embedding behavior: a new primary care challenge. Pediatrics, 2011; 127:1386-91.

10. Pereira T, Pereira S. An unusual object in the root canal of a primary tooth - a case report. Int J Paediatr Dent, 2013; 23:470-2. 